\title{
The distances measurement problem for an underwater robotic swarm: a semi-experimental trial, using power LEDs, in unknown sea water conditions
}

Received: 14 February 2020 / Accepted: 26 August 2020

(c) The Author(s) 2020

\begin{abstract}
In a preceding paper, we have showed as swarm robotics displacement can be related to the deformation of a continuum material, discretized by a lattice network representing the swarm. To reach this aim, it is fundamental to know the swarm configuration, i.e., its shape; this can be computed from the knowledge of the relative distances between its elements and it is studied as a geometry distances problem. Typically, ultrasonic devices are employed to measure the distances. We propose a method based on light signal exchanged between the machines and the computing of the unknown water adsorption coefficient and distance. Aim of this paper is, therefore, to measure distances between underwater elements of the swarm using cheap power LEDs as light source and photodiode as receiver. The receiving photodiode produces a current we can correlate with distance and water adsorption coefficient; we can be able to estimate the two unknown parameters by moving the robots and stressing the emission conditions of the LED diode. Actual work is based on a previous paper where we stressed work conditions of a power LED in shallow water to change its emission characteristics; now, using these results, we can now perform a set of measurements leading to the knowledge of distances $d$ and adsorption coefficient $a(\lambda)$. The method we propose here can be a possible support to traditional ultrasonic devices
\end{abstract}

Keywords Power LEDs · Underwater robotic swarm · Distance measurement - Geometry distance problem

\section{Introduction}

A robotic swarm presents many advantages with respect to a single autonomous underwater vehicle (AUV). Some of them are in the increase of reliability by redundancy and in the parallel working [1]; in fact, the lack of one member can be easily managed by redistributing the job among the others members like, in natural environmental, as usually done by the bees [2]. Moreover, it could limit the use of the expensive surface ships during the deployment phase, taking advantage of the parallel exploration to shorten times and having many other advantages $[3,4]$. The human operator has the possibility to examine an object concurrently from more than one point of view (in general meaning, i.e., with many sensors working from different positions), leading to a better perception of the surrounding environmental and a deeper understanding of the context. A swarm has to interact with a human operator as a single object, without the problem of controlling a large number of individuals; job sharing between the elements is an internal swarm task. This has the meaning to reveal one disadvantage of the swarm: The control system needs one more layer to translate the commands from the operator to the swarm, interfaced as a whole creature, to its constituent elements. A swarm is able to perform tasks in a more fast and robust way with respect to a single machine, but the most important feature, we want

Communicated by Luca Placidi and Ivan Giorgio.

R. dell'Erba $(\varangle)$

ENEA Technical Unit Technologies for Energy and Industry - Robotics Laboratory, Rome, Italy

E-mail: ramiro.dellerba@enea.it 
to underline, is its capability to span communication from out of the surface into the water. It allows us to realize a quasi-real-time communication and to interact with the underwater system using a remote console that can be locate on the coast. Practically, we can realize a multi-hop network with variable geometry. This is because one element could be located on the water surface, to fix its position by GPS (localization of the whole swarm follows) and to communicate with the operator; in the meanwhile, it can distribute the received message to the other elements under the sea by other kind of modem (ultrasonic or LEDs modem). The swarm has to adapt its configuration depending on the exploration mission and communication task.

ENEA is working in robotics since a long time (1961), and underwater robotics is a key topic of our laboratory [5-7]. Some years ago (2006), we have addressed our studies from single AUV to a swarm of very low-cost cooperating robots. One of the most important tasks for an underwater vehicle is its localization into the sea; for a swarm, there is also the need to know its geometric configuration. The configuration of the swarm is achieved by flocking rules, governing the movements of the single elements. In recent times, the same rules have been used to determine the deformation of a continuous material $[8,9]$, where the single elements of the swarm correspond to the particles of a discretized material. A further extension of the problem concerning this topic can be performed by considering the interplay between swarm robotics and newly conceived materials, the so-called metamaterials. Some examples of these materials can be found in [10-14] and [11,15-17]. The knowledge of the configuration is a very important issue for many applications often depending on the assigned task that can also vary during the mission. Configuration can be computed from distance measurements [6,7], and this measure is the purpose of this paper. Usually, it is done by ultrasonic measurements, but our intention is to use absorption light coefficient as support to traditional measurements.

Acoustic communications are the standards in submarine environmental [18]. Unfortunately, reflections, fading and other phenomena make difficult and sometimes not reliable the measurements. In a swarm, the need to continuously exchange data among the nodes (its elements) to get the distances represents a considerable burden for the network operation to calculate the network configuration, also using suitable algorithms; this is because it is forced to frequent short messages that deoptimize the exploitation of the communication channel, mainly for the long times needed to switch from a message to another one [19]. A possible solution, which improves both the time allocation in the acoustical protocols and unloads the acoustical channel burden, is to couple the acoustic protocol with optical device $[20,21]$ with the intention to collect distance measurements between the robots more precise, using sensor data fusion. Optical methods are very powerful, but their performance is affected by many strongly variable parameters like salinity, turbidity, the presence of dissolved substances that change the color, and the transparency of the water in different optical bands [22]. Moreover, the amount of solar radiation, in shallow water, heavily affects the signal-to-noise ratio. Our current approach uses a mixed strategy based on the variable exploitation of the optical channel depending on the environmental conditions. In favorable conditions, the transmission protocol will freely decide which channel to adopt depending on the priority, i.e., distance-to-cover and dimension of the message itself. In less favorable conditions, the optical channel will be limited to the fundamental synchronization task, generating a light lamp that will optimize the message passing through the acoustic channel. So far, we have realized an optical modem with cheap power LEDs such that it is working together with acoustic system. It must be outlined that only dense swarms can take advantage of such an approach because only in these situations, with internal distances ranging from few meters to a maximum of $20 \mathrm{~m}$, there are the conditions suitable to use light signals for sync and measurements. Moreover, backup solutions based on the "all acoustical" approach must remain available because it is always possible to find dirty waters with no way to use light signals. The use of the LED as modem has suggested as to use it also for a distance measurement. In this paper, we work on the problem to calculate distance using optical signals of this LED diode, taking in account distance and unknown adsorption coefficient. In a previous paper, we proposed the use of a cheap power led system to support acoustic devices in localization and configuration computation of an underwater robotics swarm [7]. The system was based on light signal exchanged between the machines and used power led, of different wavelength, to calculate distances between them. The unknown water conditions, affecting the light propagation, required a local measure of the absorption function $a(\lambda)$ using a known distance. The use of the LED as modem led us to a complete characterization of its static and dynamic characteristics. For this reason, we varied the duration and voltage of the power supply to study how the emission curve varied. We investigated power supply and flash duration of the LEDs can be stressed to vary light emission spectrum. By this experience, now we are able to measure distance without the local measure of the absorption function. In this paper, we show as, modifying spectrum, we shall be able to measure $a(\lambda)$ and, consequently, the distance $d$. 


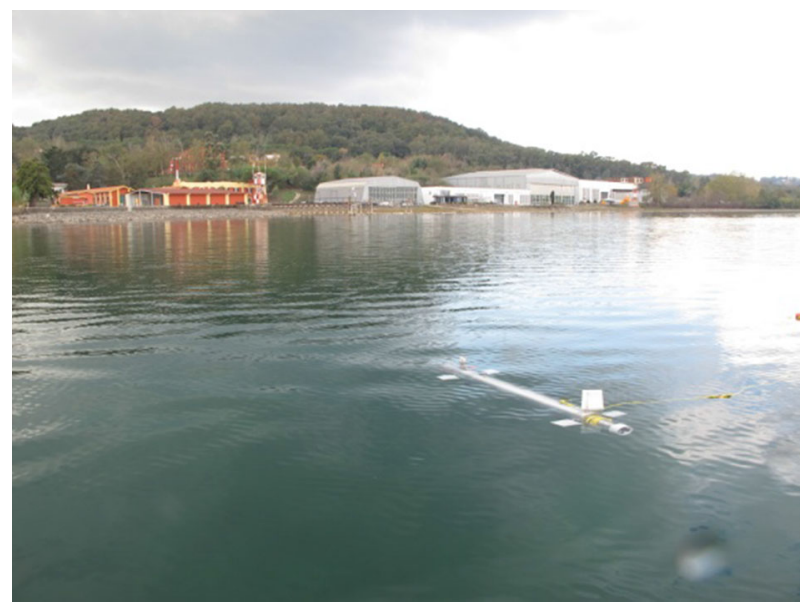

Fig. 1 Robot prototype during test in Bracciano lake

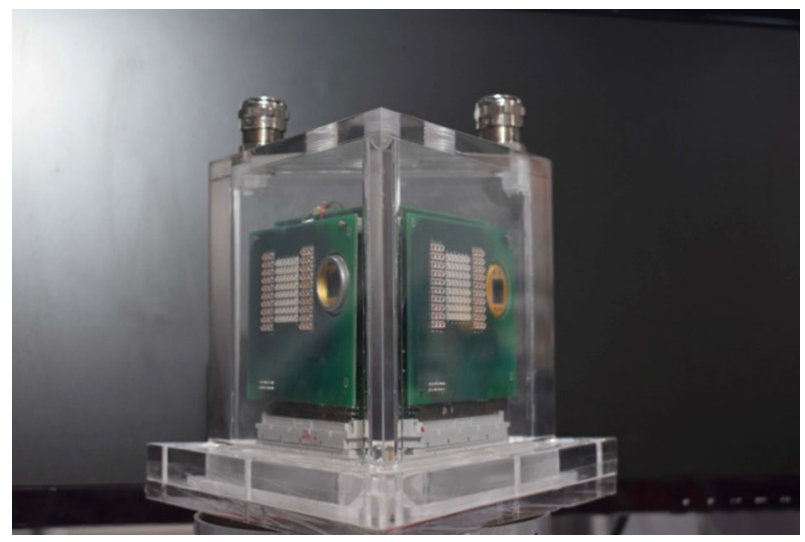

Fig. 2 Optical modem

\section{Our prototype}

In Figs. 1 and 2, the swarm element, named Venus realized in our laboratory, and the optical modem prototype are shown. The characteristics of the robot are follows: maximum depth $100 \mathrm{~m}$; maximum speed $4 \mathrm{~km} / \mathrm{h}$; weight about $20 \mathrm{~kg}$; autonomy $3 \mathrm{~h}$; dimensions are $1.20 \mathrm{~m} \times 0.20 \mathrm{~m}$ diameter. Standard sensors include a stereoscopic camera, sonar, accelerometer, compass, depth meter and hydrophones. In the optical modem, the LEDs chips and photodiode are visible; the system is repeated on three faces to cover quite $360^{\circ}$.

Note we are dealing with a system thought as to be a component of a swarm of about 20 objects. The distances between robots are between 3 and $50 \mathrm{~m}$. Therefore, the maximum distance possible between two robots is about $1000 \mathrm{~m}$, as a particular alignment case; the average value of the distances was considered about $20 \mathrm{~m}$. Dense swarm technology can be an answer in those cases where a wide acoustic bandwidth is needed, supported by other physical communication channels

\section{Semi-experimental results}

In a previous paper [7], we have used cheap power LEDs to build an optical underwater modem (see Fig. 2); to get a complete characterization of the diode characteristics, we have tried to stress power supply and flash duration to change its emission curve. In fact, LEDs technical specifications from data sheet were referred to the static work conditions and we need a more precise characterization of the diode to evaluate the possibility to use them in signaling and communication. Typical emission in working condition (27 Volts power) of a white LED is shown in Fig. 3, where the peaks, $p 1$ and $p 2$, are indicated with red points and as measurement 


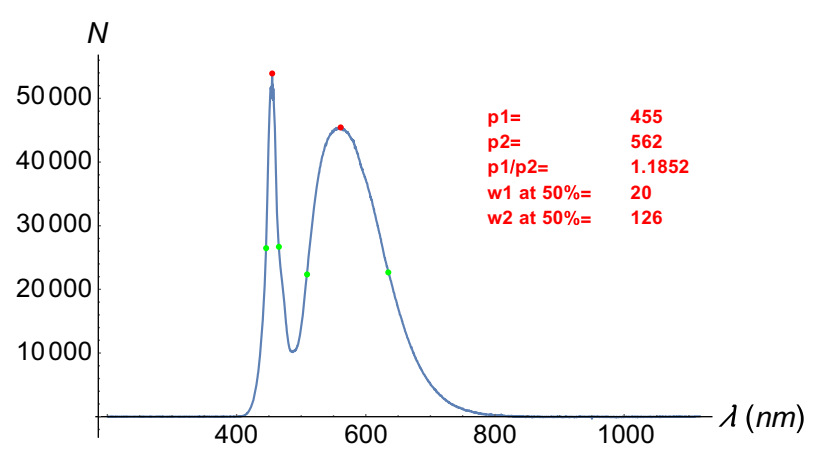

Fig. 3 White LED diode emission in normal working condition (27 Volts power); values are expressed in nanometers

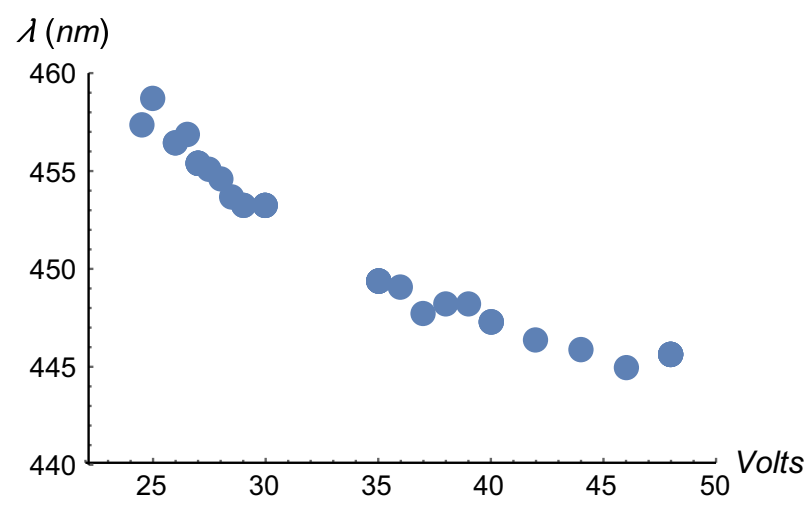

Fig. 4 White LED first peak wave length shift value vs tension

of the peak widening ( $w 1$ and $w 2$ ), the curve width at $50 \%$ of the peak height is marked with green points. Experimental result, concerning the first peak emission value vs. power supply, can be seen in Fig. 4.

The measurements have been taken with a resolution of $0.5 \mathrm{~nm}$ wavelength, and similar results have been obtained with a blue LED. The reason to attempt to vary the LED's emission characteristics is economic: Owing to the large number of swarm elements, the use of complex variable frequency system is not sustainable. Therefore, the idea was to modify the function emission of the diode by the power tension and perform distance measurements based on water light adsorption. The water adsorption is generally unknown so, in the previous work, we performed a local measurement of the attenuation coefficient by a local measure on the known distance head-tale of the robot. Unfortunately, this implies we have LEDs emission on the head of the torpedo-like robot and photodiode on the tale. This determines constructive problems of the optical modem. Our intention is to get rid of this architecture looking for another solution. The idea is that stressing LED emission we get some information useful to estimate water attenuation coefficient and, consequently, the distance. Practically, we vary the spectrum emission (Fig. 3) of the LED, varying power supply, and perform an attempt to compute absorption coefficient. In this paper, we propose a theoretical way to compute it; we have not yet experimental data but simulating how could be the collected current from the photodiode using a simple equation. Only the data concerning the emission of the LED are experimental, and this is the reason we use in the paper's title the word semi-experimental. Later, we try to match the current using some water categories, or linear combination of them, from the literature. The high number of deformed emission function in a nonlinear way allows us to avoid degeneracy solution so we get the right solution.

Light absorption by a medium, as a function of distance from the source, is a well-studied phenomenon. In a first approximation, for a spherical wave, we can assume an exponential law for the decay of the signal intensity. So, we can write

$$
I=f(\theta, \varphi) \frac{I_{0} e^{-a(\lambda) d}}{d^{2}}
$$

where $I$ is the measured intensity signal, $I_{0}$ the emitted intensity, $a(\lambda)$ the absorption function, $f(\theta, \varphi)$ a shape factor, and $d$ the distance. We do not consider here the shape factor, known for the characteristics of the 


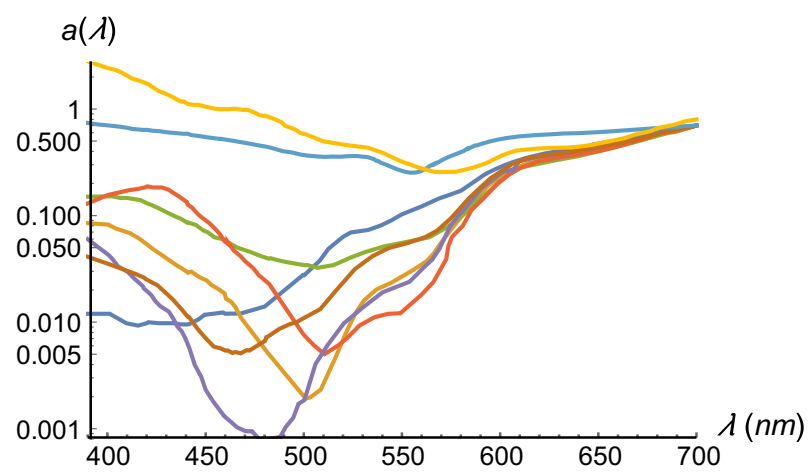

Fig. 5 Light absorption in sea water in different condition [23] from clear water to very turbid water

LED and of the photodiode leading to a modification of the spherical shape. Typically, you should consider an emission diagram of $60^{\circ} \times 60^{\circ}$; therefore, the energy is spread on this solid angle. The $a(\lambda)$, describing how the signal is attenuated as function of the wave length $\lambda$, is strongly affected by the water conditions as can be seen in Fig. 5 due to the courtesy of [23]. We are assuming that water characteristics do not vary in the volume containing the robots.

Just to have an idea about the order of magnitude of the signal attenuation in our case of interest, we consider, according to Eq. 1, a distance of $10 \mathrm{~m}$, a radius of receiving photodiode of $0,02 \mathrm{~m}$ and the case of clear water $\left(a(\lambda)\right.$ about $0.02 \mathrm{~m}^{-1}$ considering our frequency window of interest from Fig. 4); we get an attenuation of $20 \%$. We have considered $1 / 3$ as shape factor for the spherical wave because the LED emission diode is not omnidirectional. In case of turbid water $\left(a(\lambda)\right.$ about $\left.0.2 \mathrm{~m}^{-1}\right)$, the attenuation was about $90 \%$.

It is not possible to obtain both $a(\lambda)$ and $d$ by a simple measure if the intensity $I$; this is because the two objects are linked by multiplicative operator inside an exponential function so every attempt to separate them is unfruitful.

In a previous work [7], we have considered the particular case of a monochromatic LED with a sharp blue peak. In this case, we can attempt to use a nice trick, but, unfortunately, the result is affected by large error. The diode has been stressed to vary its emission peak to measure the derivative of Eq. 1 respect to $\lambda$; this gives us the product $a^{\prime}(\lambda) * d$, dividing the derivative by Eq. 1. Computing it for two, unknown, distance $d_{1}$ and $d_{2}$, we can obtain the ratio $d_{1} / d_{2}$ that is what we need. In fact, considering the ratio of Eq. 1 for the two distances, we can obtain the product $a(\lambda) * d_{1}$ as can be seen from the equation:

$$
\frac{I_{d=d_{1}}}{I_{d=d_{2}}}=\frac{d_{2}^{2} e^{-a(\lambda) d_{1}\left(1-\frac{d_{2}}{d_{1}}\right)}}{d_{1}^{2}}
$$

From this expression, we obtain $a(\lambda) * d_{1}$. Now, considering the $\log$ of Eq. 1 with $d=d_{1}$, we can obtain $a(\lambda) * d_{1}+2 \log \left(d_{1}\right)$ and consequently $d_{1}$, without any model of $a(\lambda)$.

This was very nice, but we are now using real white LEDs so we use, in this case, a semi-experimental procedure. In fact, the obtained derivative curve would be affected by many experimental errors, as usual in derivative computation, so we avoid to use the preceding method. In this paper, we have used a mixed approach where the emission curves are experimentally but combined with theoretically response of the photodiode. Therefore, we use experimental stressed emission curves of the white LED together with the, furnished by the factory, responsivity of the photodiode to calculate the collected current at different, unknown, distance. The collected current is computed by the integration of the product of the experimental emission curves, the responsivity of the photodiode and Eq. 1.

$$
\mathrm{C}=\int_{390}^{700} \mathrm{R} * \operatorname{EmLed}(\lambda) * \operatorname{Eff}(\lambda) * f(\theta, \varphi) \frac{I_{0} e^{-a(\lambda) d}}{d^{2}} \mathrm{~d} \lambda
$$

where $\operatorname{EmLed}(\lambda)$ is the emission curve of the LED (Fig. 3) in 40 different stress conditions and $E f f(\lambda)$ is the efficiency response of the photodiode obtained by the data sheet; we have also introduced a random noise $R$ of about $10 \%$. We use, as $a(\lambda)$, the eight curves of Fig. 5 to build a database. If we stress our LED emission in 40 different ways and on different unknown distances, we obtain, from the last equation, a set of curves like Figs. 6 and 7, depending on water quality. Each of the eight plot has 40 curves corresponding to the different 


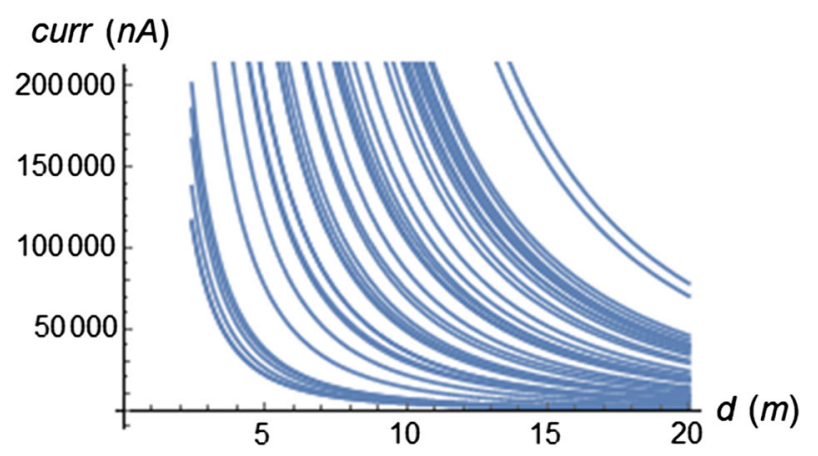

Fig. 6 Collected current by photodiode for different LED power supply vs distance in clear water $(\mathrm{w}=1)$

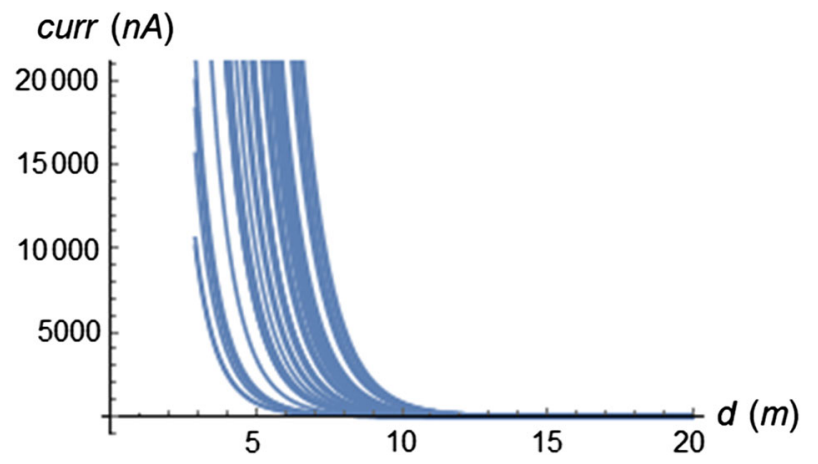

Fig. 7 Collected current by photodiode for different LED power supply vs distance in turbid water $(w=7)$

stress conditions. Therefore, we use the eight curves of Fig. 5 as preliminary database, to get eight plots like Fig. 6. Now we perform our measurements in unknown water and distances, stressing the LED in 40 different ways, and obtain 40 points set. These points are a vertical line on one of the 8 plots like Fig. 8 posed at unknown abscissa as you can see in Fig. 8; the 40 points must coincide on all the 40 curves. We repeat the operation for some other unknown distances to confirm our water quality hypothesis. We have to match our 40 points set into one of the 8 plots at a certain distance. A single measurement may present ambiguities leading to multiple choices so we repeat the measure at other distances as well as at the same one. At the end, there will be only one possible solution to match all (See Fig. 9, the green points). As "match," we mean a procedure concerning the minimization of the distances of our set of points from the curves, through optimization procedure. We are assuming the water behavior can be described by Fig. 5 or by a combination of its curves; this could be reasonable because the figure is considering a very large water conditions case. If, as usual, our water is not comprising the eight curves, we generate it from linear combination of them losing in precision.

Practically, the steps are follows: (1) simulate the collected data. We choose one of the eight kinds of water, $w$, ( 2 in our example or linear combination of them) and a distance $d$ (10.6 in our example). (2) Compute the current $C$ form the photodiode, obtaining 40 values. (3) Put these data on the plots and try to match, for each picture like Figs. 7 and 6 (or linear combination of them), the set of 40 points until we find the distance $d$.

An example can be seen in Figs. 8 and 9. Our measurements are the vertical points, and there is just one distance, in a particular kind of water, to minimize the distances of the points from the curves; we move the vertical lines to find the distance for the best match between the curves and the points.

Different cases of computing can be seen in Table 1, where the simulation for ten different distances is shown. We have performed 40 flashes for each distance and 50 measurements with random errors, stressing the LED in different conditions, for ten unknown distances. The results are quite good. Take in account that each simulated measurement is affected by a random $10 \%$ noise generate to make more realistic the computing. In Table 2, we report the results of mixed water conditions (linear combination of water of kinds 2 and 4). The results are less accurate, especially at low distances, but still useful.

Many objections can be moved to this methodology. As example, we can consider that our database can be valid only for one diode, because its chromaticism can change. Therefore, we have tried to work not with the original emission but with the ratio of the 40 emission with one of them. The results are the same, and by 


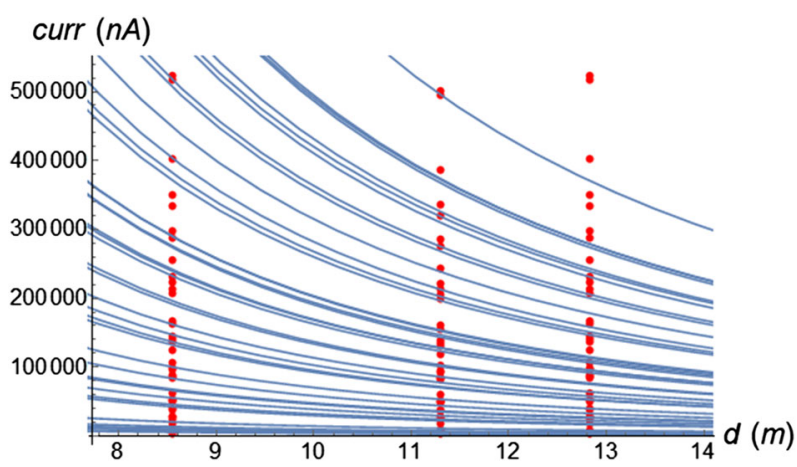

Fig. 8 Collected current by photodiode for different LED stress vs distance in water $(\mathrm{w}=4)$ and distance $=10.6 \mathrm{~m}$. The red points are our, simulated, measurements. We try to match the curves without success

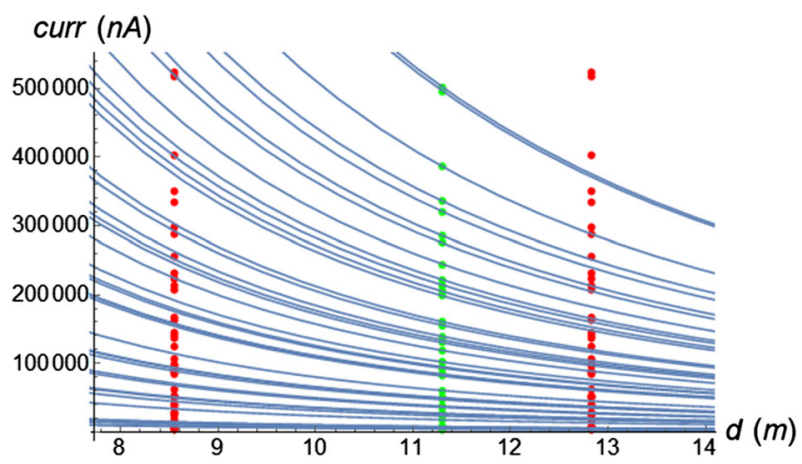

Fig. 9 Collected current by photodiode for different LED stress vs distance in water $(\mathrm{w}=2)$ and distance $=10.6 \mathrm{~m}$. The red points are our, simulated, measurements. We try to match the curves: best result is for $\mathrm{d}=11.3 \mathrm{~m}$

Table 1 Collected current by photodiode for different LED power supply vs distance in clear water $(\mathrm{w}=1)$

\begin{tabular}{lll}
\hline Real distance & Estimated distance & Standard deviation \\
\hline 0.9 & 0.9 & 0.2 \\
1.1 & 1.2 & 0.2 \\
3.5 & 3.4 & 0.1 \\
4.1 & 3.9 & 0.4 \\
4.6 & 4.4 & 0.6 \\
5.1 & 5.0 & 0.4 \\
8.3 & 7.8 & 1.3 \\
14.0 & 12.4 & 2.3 \\
16.6 & 15.1 & 3.0 \\
17.6 & 16.7 & 2.1 \\
\hline
\end{tabular}

Table 2 Estimation of the unknown distance $d$ using mix of the eight water of Fig. 5

\begin{tabular}{lll}
\hline Real distance & Estimated distance & Standard deviation \\
\hline 0.9 & 0.9 & 0.2 \\
1.1 & 1.2 & 0.2 \\
3.5 & 3.4 & 0.6 \\
4.1 & 3.8 & 0.5 \\
4.6 & 4.3 & 0.7 \\
5.1 & 4.8 & 0.7 \\
8.3 & 7.5 & 2.1 \\
14.0 & 13.4 & 2.6 \\
16.6 & 15.2 & 3.2 \\
17.6 & 16.6 & 3.1 \\
\hline
\end{tabular}




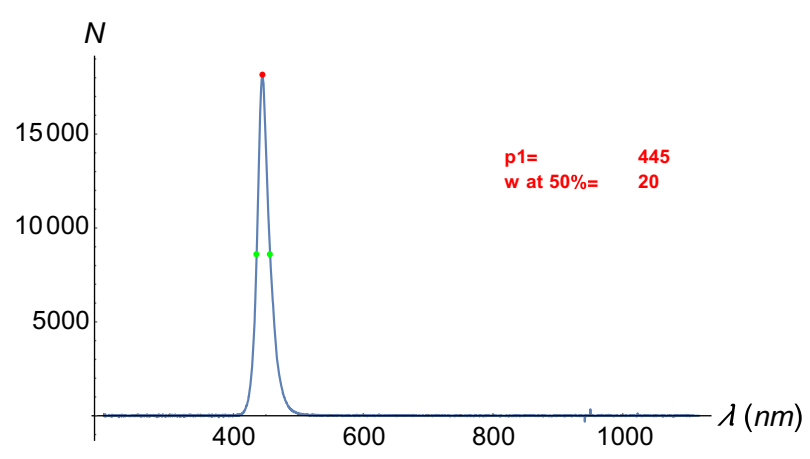

Fig. 10 Blue LED diode emission; red and green points have the same meaning of Fig. 3

this way, we avoid the problem to be linked to a particular diode. Moreover, it is possible to use more than one LED, with different frequency emission, to increase precision [7]. Working on the ratio intensity at the different frequencies, we can enhance our measurements owing to the increasing stability of the photodiode current, with respect of that of a single source. Practically, we increase the sensitivity of the signal to the measured distance, by enlarging the responsivity dynamic. It should be noted that we could obtain similar results also if we consider the same emission curve varying its amplitude because the integral in the collected current introduces a nonlinear operation (owing to the emission curve shape), and this is why we need to separate product $a(\lambda) * d$. We have tried, but it is not possible to vary only the amplitude of the emission curve. This is because, as proof by experimental data, when you change power, you obtain not only a different amplitude but also a different emission function, so we have worked directly with experimental data. The same job was performed by using a quite monochromatic blued diode (see Fig. 10) obtaining similar results with a better degree of precision owing to the sharpness of the curve.

\section{Conclusions}

We have realized an optical modem by using cheap commercial LED. Working around it, in this paper, we proposed semi-experimental results to measure distances between light source and photodiode in unknown water conditions based on adsorption coefficient. Modifying power tension, we are able to modify emission curve of the LED. The curve is stressed getting information on the water adsorption function. We speak of semiexperimental method because the emission peak is experimental, but the collected current form the photodiode is simulated by a simple equation using data furnished by the producer. The simulated collected currents are attenuated by using a database of water condition, or its linear combination; finally, varying the unknown distances, we can build a set of compatibility to identify the best matching distance $d$ and the extinction coefficient of the water $a(\lambda)$. The method could be a support for standard acoustic measurements and can be used to calculate configuration of an underwater robotic swarm that we are developing in our laboratory.

Experimental measurements are in progress to validate the concrete possibility to use this method and to reduce the error's source. This method must be considered as an iterative method whose precision is increasing with many measurements and subsequent confirmation of the previous data. These measurements must be integrated with some other source like acoustic, as usual in robot science. The use of more than one LED, with different frequency emission, suddenly increases precision. The work is in progress in our laboratory with experimental campaign into the Bracciano Lake, close to our site.

Open Access This article is licensed under a Creative Commons Attribution 4.0 International License, which permits use, sharing, adaptation, distribution and reproduction in any medium or format, as long as you give appropriate credit to the original author(s) and the source, provide a link to the Creative Commons licence, and indicate if changes were made. The images or other third party material in this article are included in the article's Creative Commons licence, unless indicated otherwise in a credit line to the material. If material is not included in the article's Creative Commons licence and your intended use is not permitted by statutory regulation or exceeds the permitted use, you will need to obtain permission directly from the copyright holder. To view a copy of this licence, visit http://creativecommons.org/licenses/by/4.0/. 
The distances measurement problem for an underwater robotic swarm

Funding Open access funding provided by Ente per le Nuove Tecnologie, l'Energia e l'Ambiente within the CRUI-CARE Agreement.

\section{References}

1. Xian-Yi, C., Shu-Qin, L., De-Shen, X.: Study of self-organization model of multiple mobile robot. Int. J. Adv. Robot. Syst. 2(3), 23 (2005)

2. Karaboga, D.: An idea based on honey bee swarm for numerical optimization. Technical report-tr06, Erciyes university, engineering faculty, computer engineering department. Available at: https://www.dmi.unict.it/mpavone/nc-cs/materiale/tr06_ 2005.pdf (2005). Access: 15-Aug-2020

3. Anderson, B., Crowell, J.: Workhorse AUV-a cost-sensible new autonomous underwater vehicle for surveys/soundings, search \& rescue, and research. In: Proc. MTSIEEE OCEANS 2005, pp. 1228-1233 (2005)

4. Shah, V.P.: Design considerations for engineering autonomous underwater vehicles. Design considerations for engineering AUVs (2007)

5. Dell'Erba, R., Moriconi, C.: Bio-inspired robotics—it. [In linea]. Available at: http://www.enea.it/it/produzione-scientifica/ edizioni-enea/2014/bio-inspirede-robotics-proceedings (2014). Accessed 15 Dec 2019

6. Dell'Erba, R.: Determination of spatial configuration of an underwater swarm with minimum data. Int. J. Adv. Robot. Syst. 12(7), 97 (2015). https://doi.org/10.5772/61035

7. Dell'Erba, R., Moriconi, C.: High power leds in localization of underwater robotics swarms. IFAC-Pap. 48(10), 117-122 (2015). https://doi.org/10.1016/j.ifacol.2015.08.118

8. Dell'Erba, R.: Swarm robotics and complex behaviour of continuum material. Contin. Mech. Thermodyn. 31, 989-1014 (2019). https://doi.org/10.1007/s00161-018-0675-1

9. Wiech, J., Eremeyev, V.A., Giorgio, E.I.: Virtual spring damper method for nonholonomic robotic swarm self-organization and leader following. Contin. Mech. Thermodyn. 30(5), 1091-1102 (2018). https://doi.org/10.1007/s00161-018-0664-4

10. Di Cosmo, F., Laudato, M., Spagnuolo, E.M.: Acoustic metamaterials based on local resonances: homogenization, optimization and applications. In: Advanced Structured Materials, pp. 247-274 (2018)

11. Barchesi, E., Spagnuolo, M., Placidi, E.L.: Mechanical metamaterials: a state of the art. Math. Mech. Solids 24(1), 212-234 (2018). https://doi.org/10.1177/1081286517735695

12. Turco, E.: Tools for the numerical solution of inverse problems in structural mechanics: review and research perspectives. Eur. J. Environ. Civ. Eng. 21(5), 509-554 (2017). https://doi.org/10.1080/19648189.2015.1134673

13. Giorgio, I., Rizzi, N.L., Turco, E.E.: Continuum modelling of pantographic sheets for out-of-plane bifurcation and vibrational analysis. Proc. R. Soc. Math. Phys. Eng. Sci. 473(2207), 20170636 (2017). https://doi.org/10.1098/rspa.2017.0636

14. Alibert, J.-J., Seppecher, P., Dell'isola, E.F.: Truss modular beams with deformation energy depending on higher displacement gradients. Math. Mech. Solids 8(1), 51-73 (2003)

15. Dell'Isola, F., et al.: Pantographic metamaterials: an example of mathematically driven design and of its technological challenges. Contin. Mech. Thermodyn. 31(4), 851-884 (2019)

16. Dell'Isola, F., et al.: Advances in pantographic structures: design, manufacturing, models, experiments and image analyses. Contin. Mech. Thermodyn. 31(4), 1231-1282 (2019)

17. Turco, E., Giorgio, I., Misra, A., Dell'Isola, E.F.: King post truss as a motif for internal structure of (meta) material with controlled elastic properties. R. Soc. Open Sci. 4(10), 171153 (2017)

18. Kottege, N., Zimmer, E.U.R.: Acoustical methods for Azimuth, range and heading estimation in underwater swarms. J. Acoust. Soc. Am. 123(5), 3007 (2008). https://doi.org/10.1121/1.2932587

19. Schill, F.S.: Distributed communication in swarms of autonomous underwater vehicles. The Australian National University. Available at: http://schillnet.scinamics.com/studies/Publications/Schill.2007.PhDThesis.pdf (2007). Accessed 15 Aug 2020

20. Han, S., Noh, Y., Liang, R., Chen, R., Cheng, Y.-J., Gerla, E.M.: Evaluation of underwater optical-acoustic hybrid network. Commun. China 11(5), 49-59 (2014)

21. Farr, N., Bowen, A., Ware, J., Pontbriand, C., Tivey, E.M.: An integrated, underwater optical/acoustic communications system. In: OCEANS 2010 IEEE-Sydney. pp. 1-6 (2010)

22. Anguita, D., Brizzolara, D., Parodi, G., Hu, Q.: Optical wireless underwater communication for AUV: preliminary simulation and experimental results. In: OCEANS 2011 IEEE, Spain, Santander. pp. 1-5 (2011). https://doi.org/10.1109/Oceans-Spain. 2011.6003598

23. Bogdan, W., Jerzi, E.D.: Light Absorption in Sea Water. Springer, Berlin (2007)

Publisher's Note Springer Nature remains neutral with regard to jurisdictional claims in published maps and institutional affiliations. 